\title{
Resection and/ or Thermal Ablation for Recurrent Biliary Tract Cancer
}

\author{
Ikuta S*, Aihara T, Nakajima T and Yamanaka N \\ Department of Surgery, Meiwa Hospital, J apan \\ *Corresponding author: Shinichi Ikuta, Department \\ of Surgery, Meiwa Hospital, Agenaruo 4-31, Nishinomiya, \\ Hyogo 663-8186, J apan
}

Received: January 17, 2017; Accepted: February 21, 2017; Published: February 24, 2017

\begin{abstract}
Aim: Repeat resection of recurrent Biliary Tract Cancer (BTC) is possible in a limited number of patients, with thermal ablation being an alternative for small hepatic recurrences. The aim of this study was to investigate the beneficial effect of these interventions on survival in patients with recurrent BTC.
\end{abstract}

Methods: One hundred and six recurrent BTC patients were divided a group of patients who received intervention (surgical resection and/or thermal ablation) for recurrence (group 1, $\mathrm{n}=26$ ) and another group who did not (group 2, $n=80$ ). The outcome of both groups was investigated retrospectively.

Results: There were no significant differences between the two groups with respect to demographic data, underlying pathology, primary tumor stage and initial disease-free interval. In group 1, hepatic recurrence was most common $(n=24)$, followed by locoregional recurrence $(n=13)$ and peritoneal seeding $(n=7)$. A total of 41 interventions were performed in group 1. Post-recurrence survival was significantly better in group 1 than in group 2 (median: 19.4 vs. 10.5 months; $p<0.05$ ). An initial disease-free interval $\geq 2$ years, absence of macroscopic residual tumor after initial intervention and two or more interventions were significant predictors of better survival after intervention. Overall survival after resection of the primary tumor was more favorable in group 1 than group 2 , but the difference was not significant (median 44.3 vs. 30.7 months; $p=0.07$ ).

Conclusion: Repeat resection and/or thermal ablation can be valuable therapeutic options that achieve significant prolongation of post-recurrence survival in selected patients with recurrent BTC.

Keywords: Secondary surgery; Thermal ablation; Radio frequency ablation; Biliary tract cancer; Recurrence; Recurrent BTC

\section{Introduction}

Biliary Tract Cancer (BTC), including cholangiocarcinoma, gallbladder carcinoma and ampullary carcinoma, has a poor prognosis with a 5 -year survival rate of only $20.2 \%$ in Japan [1]. Complete surgical resection is currently the only curative therapy for BTC. However, even if radical resection is performed, early recurrence (either locoregional or metastatic) occurs in a considerable proportion of patients. Management of recurrent BTC is challenging because the disease is often aggressive and there is a lack of effective therapy. Although chemotherapy or radiotherapy is usually given as palliative treatment to improve symptoms and extend survival [2-4], the results are unsatisfactory. A few studies have explored the outcome of secondary surgery or thermal ablation for recurrent BTC [5-8], but there is no consensus regarding the role, indications and limitations of such treatment. In this retrospective study, we investigated the influence of resection and/or thermal ablation on survival in patients with recurrent BTC.

\section{Patients and Methods}

Between January 2003 and December 2015, 196 consecutive patients with BTC (including intrahepatic cholangiocarcinoma) underwent surgical resection at our institute. Of the 173 patients who had R0 or R1 resection, 106 (61\%) developed recurrence during a mean follow-up period of 39.9 months (range: 1-155 months). Among them, 26 patients underwent repeat resection and/or thermal ablation to treat the recurrent tumor (group 1), whereas the remaining 80 patients did not (group 2). In group 1, imaging studies performed following treatment-whether primary tumor resection, post-operative adjuvant chemotherapy or palliative chemotherapy for recurrent disease-revealed that the recurrent tumor was potentially resectable without any signs of aggressive growth or widespread disease. Thermal ablation (microwave or Radiofrequency Ablation: RFA) was performed alone or in combination with hepatic resection for small $(\leq 3 \mathrm{~cm})$ central hepatic recurrences. Patients who were poor candidates for surgery were often treated by percutaneous RFA. When thermal ablation was performed in a patient with entero-biliary anastomosis, oral prophylaxis with levofloxacin (500 mg daily) was started from 5 days before the procedure and continued for a total of 7 days to reduce the risk of liver abscess. Survival outcomes of each group were investigated by reviewing the medical records.

\section{Statistics}

Categorical variables were compared by the chi-square test or Fisher's exact test. Survival analysis was performed using KaplanMeier estimation and the log-rank test. Prognostic factors were identified by using multivariate Cox proportional hazards models. All statistical analyses were performed with EZR (Saitama Medical 
Table 1: Characteristics of the two groups.

\begin{tabular}{|c|c|c|c|}
\hline & group $1(n=26)$ & group $2(n=80)$ & $p$ \\
\hline Age, median (yr) & 63.5 & 71 & 0.07 \\
\hline Gender (M:F) & $13: 13$ & $54: 26: 00$ & 0.16 \\
\hline \multicolumn{4}{|l|}{ Primary cancer, n (\%) } \\
\hline Intrahepatic & $12(46)$ & $13(16)$ & \multirow{5}{*}{0.06} \\
\hline Perihilar & $4(15)$ & $17(21)$ & \\
\hline Distal & $5(19)$ & $20(25)$ & \\
\hline Ampulla & $1(4)$ & $6(8)$ & \\
\hline Gallbladder & $4(15)$ & $24(30)$ & \\
\hline \multicolumn{4}{|l|}{ Tumor staging, n (\%) } \\
\hline I $(\mathrm{IA}, \mathrm{IB})$ & $0(0)$ & $3(4)$ & \multirow{4}{*}{0.15} \\
\hline II (IIA, IIB) & $13(50)$ & $35(44)$ & \\
\hline III (IIIA, IIIB) & $9(35)$ & $27(34)$ & \\
\hline IV (IVA, IVB) & $4(15)$ & 15(19) & \\
\hline R0/R1, n & $24 / 2$ & $65 / 15$ & 0.18 \\
\hline \multicolumn{4}{|l|}{ Types of recurrence, $n$} \\
\hline Hepatic & 24 & 52 & \multirow{8}{*}{0.66} \\
\hline \multicolumn{4}{|l|}{ Locoregional } \\
\hline Lymph node or perineural & 7 & 34 & \\
\hline Bile duct & 6 & 8 & \\
\hline Peritoneal seeding & 7 & 14 & \\
\hline \multicolumn{3}{|l|}{ Other distant metastasis } & \\
\hline Adrenal gland & 3 & 0 & \\
\hline Lung & 1 & 4 & \\
\hline Bone & 0 & 3 & \\
\hline Adjuvant chemotherapy, n (\%) & 18(69) & $53(66)$ & 0.78 \\
\hline IDFI, median (month) & 13.2 & 9.5 & 0.22 \\
\hline Palliative chemotherapy, n (\%) & $23(88)$ & $67(84)$ & 0.76 \\
\hline
\end{tabular}

IDFI: Initial Disease-Free Interval

Center, Jichi Medical University, Saitama, Japan), which is a graphical user interface for $\mathrm{R}$ (The R Foundation for Statistical Computing, Vienna, Austria) and $p<0.05$ was considered significant.

\section{Results}

\section{Characteristics of the two groups}

There were no significant differences between the groups with respect to demographic data, underlying pathology and primary tumor stage. The proportion of patients receiving chemotherapy also did not differ between the groups. Recurrence was classified as hepatic recurrence, locoregional recurrence (lymph node metastasis, perineural invasion or remnant bile duct recurrence), peritoneal seeding or other distant metastasis. In group 1, the most common type of recurrence was hepatic recurrence $(n=24)$, followed by locoregional recurrence $(n=13)$ and peritoneal seeding $(n=7)$. The Initial Disease-Free Interval (IDFI) after surgery for primary BTC was 13.2 months in group 1 and 9.5 months in group 2, with no significant difference of IDFI between the two groups (Table 1).

\section{Treatment of recurrence}

The median time from detection of recurrence to treatment was
Table 2: Treatment of recurrence in group 1.

\begin{tabular}{|c|c|}
\hline Procedures & $\mathrm{n}$ \\
\hline Hepatic resection $\mathrm{Hr} 2 / \mathrm{Hr} 1 / \mathrm{HrS} / \mathrm{HrO}$ & $3 / 2 / 1 / 9$ \\
\hline Thermal ablation & 15 \\
\hline Lymphadenectomy & 7 \\
\hline Resection of the peritoneal seeding & 7 \\
\hline Right adrenalectomy & 3 \\
\hline PpPD & 1 \\
\hline Lung resection & 1 \\
\hline Combined & 8 \\
\hline R0/R1/R2 & $30 / 7 / 12$ \\
\hline Morbidity (Clavien-Dindo $\geq$ IIla) & 5 \\
\hline Mortality & 0 \\
\hline
\end{tabular}

$\mathrm{Hr}$ : liver bisegmentectomy; $\mathrm{Hr} 1$ : segmentectomy; $\mathrm{HrS}$ : Couinaud's segmentectomy; HrO: limited resection; PpPD: Pylorus-preserving pancreatoduodenectomy
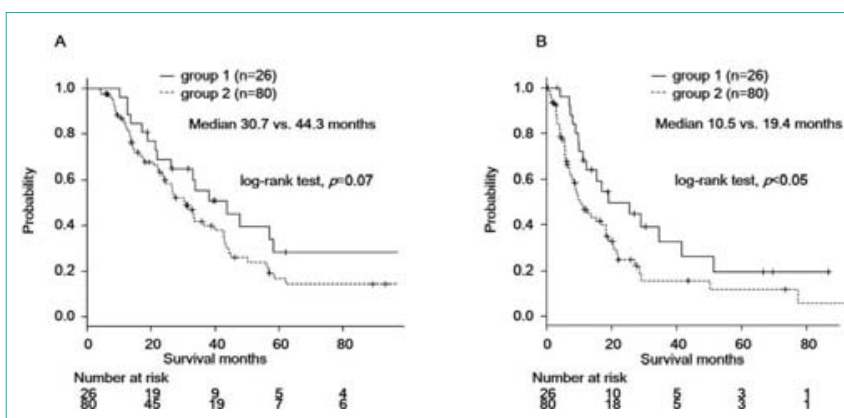

Figure 1: Survival after surgery for the primary cancer $(A)$ and survival after recurrence (B) in group 1 and group 2 .

2.5 months (range: 1-11 months). A total of 41 interventions were performed for recurrent disease in group 1, with 17 patients receiving one intervention, 5 patients receiving two, 3 patients receiving three and 1 patient receiving five. The procedures and results are listed in (Table 2). Twelve of the 41 interventions ended as $\mathrm{R} 2$ resection, including five patients who were found to have unresectable disease with diffuse peritoneal seeding at operation. In the remaining 7 patients, the local recurrence could not be radically resected. The morbidity rate (Clavien-Dindo classification IIIa or higher) and mortality rate were $12 \%$ and $0 \%$ respectively. None of the patients developed a hepatic abscess requiring percutaneous drainage after thermal ablation.

\section{Survival}

The 1- and 3-year Post-Recurrence Survival (PRS) rates were respectively $68.0 \%$ and $32.6 \%$ in group 1 versus $45.9 \%$ and $15.4 \%$ in group 2. Median PRS time was 19.4 months for group 1 and 10.5 months for group 2 , showing a significant difference $(p<0.05)$. The 3 -year and 5-year Overall Survival (OS) rates after surgery for primary BTC were respectively $55.4 \%$ and $28.2 \%$ in group 1 versus $41.1 \%$ and $16.7 \%$ in group 2. Median OS time was longer in group 1 than in group 2 ( $44.3 v s$. 30.7 months; $p=0.07$ ), although the difference was not significant (Figure 1). Multivariate analysis identified intervention for recurrent BTC (HR=1.761, 95\% CI 1.013 to 3.062; $p=0.045)$ and $\mathrm{R} 0$ resection for the primary tumor $(\mathrm{HR}=1.926,95 \%$ CI 1.008 to 3.679 ; 
Table 3: Multivariate analysis of factors predicting survival after intervention in group 1.

\begin{tabular}{|c|c|c|c|c|c|c|}
\hline \multirow{2}{*}{ Variables } & \multicolumn{2}{|c|}{ Characteristics } & \multirow{2}{*}{ HR } & \multicolumn{2}{|c|}{$95 \% \mathrm{Cl}$} & \multirow{2}{*}{$p$} \\
\hline & Unfavorable & Favorable & & Lower & Upper & \\
\hline IDFI & $|D F|<2 y$ & $|D F| \geq 2 y$ & 0.255 & 0.074 & 0.877 & 0.03 \\
\hline Residual tumor & $\mathrm{R} 2$ & $\mathrm{R} 0 / \mathrm{R} 1$ & 0.142 & 0.03 & 0.671 & 0.01 \\
\hline Primary cancer stage & III/IV & $\mathrm{I} / \mathrm{II}$ & 0.655 & 0.178 & 2.411 & 0.52 \\
\hline Frequency of intervention & once & $\begin{array}{c}\text { twice } \\
\text { or more }\end{array}$ & 0.144 & 0.034 & 0.604 & $<0.01$ \\
\hline Peritoneal seeding & present & absent & 0.583 & 0.138 & 2.458 & 0.46 \\
\hline
\end{tabular}

IDFI: Initial Disease-Free Interval

$p=0.047$ ) as independent prognostic factors on PRS.

\section{Prognostic factors in group 1 after intervention}

To investigate whether certain subgroups of patients would benefit more from intervention, prognostic factors affecting survival after intervention in group 1 were evaluated by multivariate analysis. It was found that patients with a longer IDFI ( $\geq 2$ years) were more likely to benefit from intervention compared to patients showing recurrence within 2 years (median: 40.0 vs. 10.0 months; $p<0.05$ ). In addition, absence of macroscopic residual tumor after the initial intervention (median: 40.0 vs. 8.0 months; $p<0.01$ ) and two or more interventions (median: 33.0 vs.10.0 months, $p<0.05$ ) were identified as independent prognostic factors (Table 3 ).

\section{Discussion}

In 2010, a British randomized phase III trial established Gemcitabine/Cisplatin (Gem/CDDP) as a standard chemotherapy regimen for advanced BTC [2]. The efficacy of Gem/CDDP therapy was also confirmed in a study of Japanese patients [3]. However, the median OS obtained with Gem/CDDP in these trials was only about 11 months, which was similar to median PRS (10.5 months) in our group 2 patients. In the present study, group 1 had significantly better PRS than group 2, indicating that resection and/or thermal ablation offers a survival benefit for selected patients compared with chemotherapy alone or best supportive care.

To our knowledge, there have only been three retrospective cohort studies on surgical treatment of recurrent BTC. Song, et al. [5] investigated 27 patients undergoing surgery for recurrent cholangiocarcinoma and found that the PRS rate was significantly higher in surgically treated patients compared with patients who were treated non-surgically (median PRS:18.9 vs. 7.7 months; $p=0.001$ ). A relatively large-scale study was performed by Takahashi, et al. [6], which revealed that both OS ( $35 v s .11 \%$ at 5 years; $p<0.001)$ and PRS ( $37 v$ s. $3 \%$ at 3 years; $p<0.001$ ) were significantly better in 74 recurrent BTC patients undergoing surgery than in 532 patients without surgery. Furthermore, Noji, et al. [7] performed surgery for recurrence in 27 patients with gallbladder carcinoma and extra hepatic cholangiocarcinoma and concluded that it was feasible and offered longer survival for selected patients (median OS time: $21.6 \mathrm{vs}$. 9.5 months; $p<0.01)$.

Later recurrence is associated with improved survival in patients with various malignancies, possibly due to the natural history of slower-growing tumors. Analysis of 30 patients undergoing surgery for recurrent pancreatic cancer showed that patients with a longer interval from resection to recurrence ( $>9$ months) were more likely to benefit from re-resection than patients who developed recurrence within 9 months (median survival time: 17.0 vs. 7.4 months; $p=0.004$ ) [9]. Our study showed that IDFI ( $\geq 2$ years $v$ s. $<2$ years) had a significant influence on survival after intervention for recurrence, a finding that was consistent with one of the above-mentioned studies focusing on surgical treatment of recurrent BTC [6]. We also identified some other prognostic factors after intervention. In patients with peritoneal seeding, surgical intervention frequently ended as R2 resection and this was a significant poor prognostic factor in group 1, suggesting that absence of macroscopic residual tumor should be the goal of intervention to obtain a favorable outcome. Indeed, apart from 1 patient who survived for 33 months, the patients with peritoneal seeding did not survive for longer than 1 year after secondary surgery. This might be associated with previous studies showing that survival of patients after resection of chest or abdominal wall recurrence was worse than after surgery for other recurrences [6]. Because even localized peritoneal seeding might be an indicator of future widespread dissemination, surgical indications for patients with peritoneal seeding should be considered carefully.

Recent advances have made local ablative treatment for liver tumors more accessible. RFA has several potential advantages over surgery, including higher repeatability, greater tolerability, lower cost and lower complication rates [10]. We used thermal ablation alone or in combination with surgical resection to treat hepatic recurrence. In particular, patients with small central or multifocal liver lesions and patients who were poor candidates for surgery often received thermal ablation. In patients with entero-biliary anastomosis, the risk of ablation-induced liver abscess could be reduced by antibiotic prophylaxis. Several previous studies have found that thermal ablation achieves similar local tumor control to that obtained with hepatic resection in patients with colorectal liver metastasis [11], as well as patients with hepatocellular carcinoma [12]. Zhang, et al. [8] reported that repeat liver resection and thermal ablation both have similar overall efficacy for recurrent intrahepatic cholangiocarcinoma. It seems that thermal ablation represents a promising therapeutic option, even for hepatic recurrence of BTC.

The limitations of this study include a small sample size and single-center design. Another important limitation is inherent selection bias due to the retrospective nature of the study. Patients in group 1 were candidates for intervention because they had adequate organ function, a resectable recurrence, or hepatic recurrence for which thermal ablation was applicable, while patients in group 2 did not necessarily have such favorable factors. Other factors, such as frailty, disability and malnutrition, may also have affected treatment 
choice. Though this selection bias could interfere with drawing strong conclusions, the potential benefits of intervention remain intriguing.

\section{Conclusion}

Resection and/or thermal ablation of recurrent BTC may improve PRS in a small, but significant, group of patients. Given the limited usefulness of chemotherapy or radiotherapy, these interventions are important options that should be pursued, especially for late and macroscopically resectable recurrence. Repeat intervention should also be the treatment of choice for re-recurrence when possible Treatment of recurrent BTC requires a multidisciplinary approach and careful selection of the most appropriate strategy for each patient. More studies are needed to further define the role of secondary surgery and to clarify the optimal combinations of different therapeutic options.

\section{References}

1. Matsuda T, Ajiki W, Marugame T, loka A, Tsukuma H, Sobue T. Research Group of Population-Based Cancer Registries of Japan. Population-based survival of cancer patients diagnosed between 1993 and 1999 in Japan: a chronological and international comparative study. Jpn J Clin Oncol. 2011; 41: 40-51.

2. Valle $\mathrm{J}$, Wasan $\mathrm{H}$, Palmer $\mathrm{DH}$, Cunningham $\mathrm{D}$, Anthoney A, Maraveyas A, et al. Cisplatin plus gemcitabine versus gemcitabine for biliary tract cancer. $\mathrm{N}$ Engl J Med. 2010; 362: 1273-1281.

3. Okusaka T, Nakachi K, Fukutomi A, Mizuno N, Ohkawa S, Funakoshi A, et al. Gemcitabine alone or in combination with cisplatin in patients with biliary tract cancer: a comparative multicentre study in Japan. Br J Cancer. 2010; 103: $469-474$.
4. Jung DH, Kim MS, Cho CK, Yoo HJ, Jang WI, Seo YS, et al. Outcomes of stereotactic body radiotherapy for unresectable primary or recurrent cholangio carcinoma. Radiat Oncol J. 2014; 32: 163-169.

5. Song SC, Heo JS, Choi DW, Choi SH, Kim WS, Kim MJ. Survival benefits of surgical resection in recurrent cholangio carcinoma. J Korean Surg Soc. 2011; 81: 187-194.

6. Takahashi $Y$, Ebata T, Yokoyama $Y$, Igami T, Sugawara G, Mizuno T, et al Surgery for recurrent biliary tract cancer: A single-center experience with 74 consecutive resections. Ann Surg. 2015; 262: 121-129.

7. Noji T, Tsuchikawa T, Mizota T, Okamura K, Nakamura T, Tamoto E, et al Surgery for recurrent biliary carcinoma: results for 27 recurrent cases. World J Surg Oncol. 2015; 27: 13-82.

8. Zhang SJ, Hu P, Wang N, Shen Q, Sun AX, Kuang M, et al. Thermal ablation versus repeated hepatic resection for recurrent intrahepatic cholangio carcinoma. Ann Surg Oncol. 2013; 20: 3596-3602.

9. Kleeff J, Reiser C, Hinz U, Bachmann J, Debus J, Jaeger D, et al. Surgery for recurrent pancreatic ductal adenocarcinoma. Ann Surg. 2007; 245: 566-572.

10. Liu PH, Hsu CY, Hsia CY, Lee YH, Huang YH, Chiou YY, et al. Surgical resection versus radiofrequency ablation for single hepatocellular carcinoma $\leq 2 \mathrm{~cm}$ in a propensity score model. Ann Surg. 2016; 263: 538-545.

11. Van der Pool AE, Lalmahomed ZS, De Wilt JH, Eggermont AM, ljzermans JM, Verhoef C. Local treatment for recurrent colorectal hepatic metastases after partial hepatectomy. J Gastrointest Surg. 2009; 13: 890-895.

12. Chan AC, Poon RT, Cheung TT, Chok KS, Chan SC, Fan ST, et al. Survival analysis of re-resection versus radiofrequency ablation for intrahepatic recurrence after hepatectomy for hepatocellular carcinoma. World J Surg. 2012; 36: 151-156
Austin J Surg - Volume 4 Issue 2 - 2017

ISSN : 2381-9030 | www.austinpublishing group.com

Ikuta et al. (C) All rights are reserved
Citation: Ikuta S, Aihara T, Nakajima T and Yamanaka N. Resection and/or Thermal Ablation for Recurrent Biliary Tract Cancer. Austin J Surg. 2017; 4(2): 1098 